\title{
DECODING SATELLITE IMAGES OF EARTHQUAKES, PART I
}

\section{Saumik Dana ${ }^{1, *}$}

\author{
${ }^{1}$ University of Southern California, Los Angeles, CA 90089 \\ *Address all correspondence to: Saumik Dana, E-mail: sdana@usc.edu
}

This document is part I of a series of documents providing a lowdown on image processing in the context of understanding satellite images of earthquakes

KEY WORDS: Earthquakes, imaging

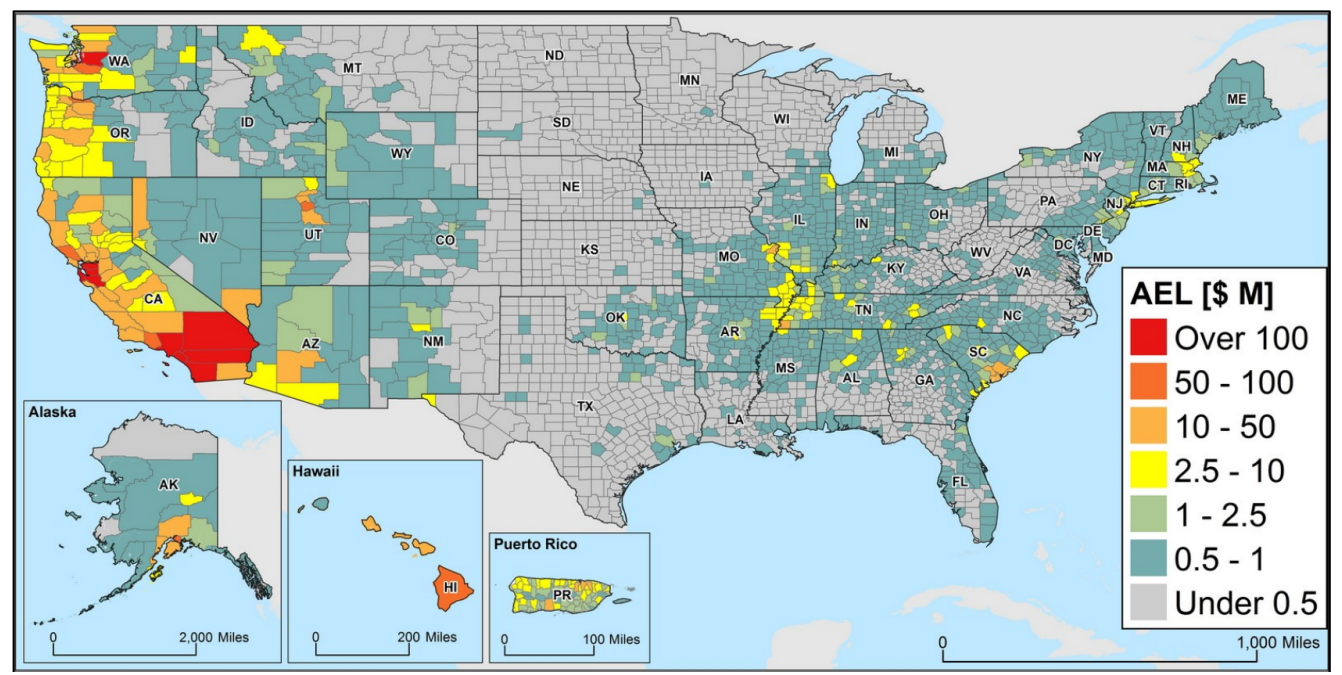

FIG. 1: Annualized Earthquake Losses (AEL: estimated long-term value of earthquake losses to the general building stock) by County. Sources: (1) https://www.usgs.gov/news/usgs-collaborates-fema-national-earthquake-loss-estimate and (2) https://www.fema.gov/hazus. The analysis yielded an estimate of the national AEL of $\$ 6.1$ billion per year. Loss estimates based on the best science and engineering available (during 2016-2017). The study only addresses direct economic losses to buildings, and does not cover damage and losses to critical facilities, transportation and utility lifelines or indirect economic losses.

Ground motion and failure due to earthquakes lead to huge losses to the tune of billions of dollars annually in the United States (see Fig. 1). Detecting and cataloging these earthquakes are key to understanding their causes (natural or human-induced (Dana (2018, 2019); Dana et al. (2018, 2020); Dana and Jha (2021); Dana and Wheeler (2018a,b, 2019); Dana et al. (2021); Foulger et al. (2018))) and, ultimately, to mitigating the seismic risk. There are in essence, two approaches

$\checkmark$ Template matching i.e. signal processing, with the input being the seismogram signals. This approach has a historic precendence, and is most often used due to its ubiquity (Valentine and Trampert (2012); Yoon et al. (2015))

$\checkmark$ Image processing i.e. Computer vision, with the input being the satellite images of earthquakes. This approach has been taking shape this decade since the advent of GPU computable neural network arithmetic computations 
(Krizhevsky et al. (2012)), while leveraging all the progress in the realm of medical image processing (McCann et al. (2017))

There are two mathematical concepts that are common to practically all signal and image processing techniques

$\checkmark$ Support vector machines

$\checkmark$ Principal component analysis

\section{SUPPORT VECTOR MACHINES}

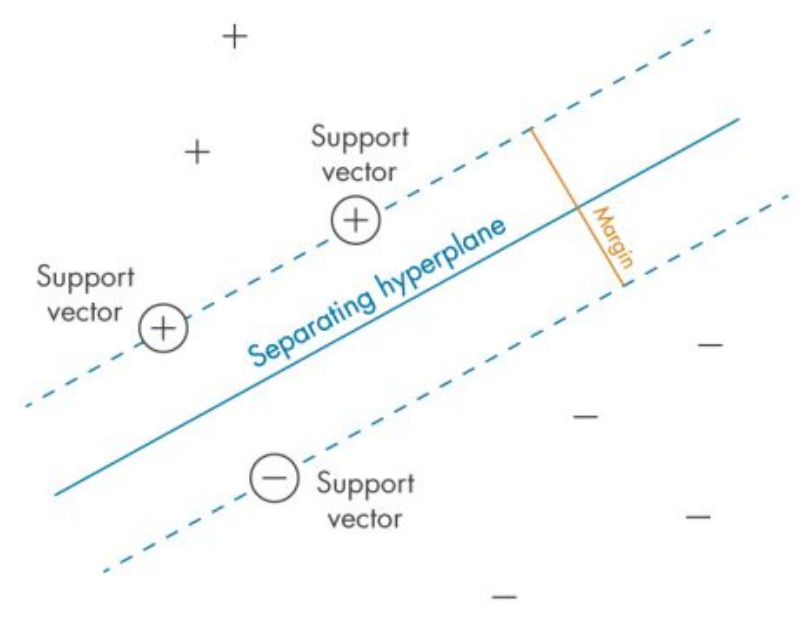

FIG. 2: Support vector "machines”. Source: mathworks.com

Support vector "machine" is a misleading term: it's a maximization problem and a classification technique. The basic idea is to find a hyperplane which separates the d-dimensional data perfectly into its two classes (Burges (1998); Cortes and Vapnik (1995), see also Fig. 2). We are given $l$ training examples $\left\{x_{i}, y_{i}\right\}, i=1, \cdots, l$, where each example has $d$ inputs $\left(x_{i} \in R^{d}\right)$, and a class label with one of two values $\left(y_{i} \in\{-1,1\}\right)$. Now, all hyperplanes in $R^{d}$ are parameterized by a vector $(\mathrm{w})$, and a constant $(b)$, expressed in the equation

$$
\mathbf{w} \cdot \mathbf{x}+b=0
$$

So we define the canonical hyperplane to be that which separates the data from the hyperplane by a "distance" of at least 1 . That is, we consider those that satisfy:

$$
y_{i}\left(\mathbf{x}_{\mathbf{i}} \cdot \mathbf{w}+b\right) \geq 1 \quad \forall i
$$

To obtain the geometric distance from the hyperplane to a data point, we must normalize by the magnitude of $\mathrm{w}$. This distance is simply:

$$
d\left((\mathbf{w}, b), \mathbf{x}_{i}\right)=\frac{y_{i}\left(\mathbf{x}_{\mathbf{i}} \cdot \mathbf{w}+b\right)}{\|\mathbf{w}\|} \geq \frac{1}{\|\mathbf{w}\|}
$$

From the equation we see this is accomplished by minimizing $\|\mathbf{w}\|$ (subject to the distance constraints). The main method of doing this is with Lagrange multipliers. The problem is eventually transformed into:

$$
\begin{array}{cc}
\text { minimize: } & W(\alpha)=-\alpha^{T} 1+\frac{1}{2} \alpha^{T} H \alpha \\
\text { subject to: } & \alpha^{T} y=0 \\
& 0 \leq \alpha \leq C 1
\end{array}
$$


where $\alpha$ is the vector of $l$ non-negative Lagrange multipliers to be determined, $C$ is a constant and $(H)_{i j}=y_{i} y_{j}\left(\mathbf{x}_{i}\right.$. $\mathbf{x}_{j}$ ). In addition, from the derivation of these equations, it was seen that the optimal hyperplane can be written as:

$$
\mathbf{w}=\sum_{i} \alpha_{i} y_{i} \mathbf{x}_{i}
$$

That is, the vector $w$ is just a linear combination of the training examples. Since example data is often not linearly separable, SVM's introduce the notion of a "kernel induced feature space" which casts the data into a higher dimensional space where the data is separable. There is a way to "pre-process" the data in such a way that the problem is transformed into one of finding a simple hyperplane. To do this, we define a mapping $\mathbf{z}=\phi(\mathbf{x})$ that transforms the $d$ dimensional input vector $\mathbf{x}$ into a (usually higher) $d^{\prime}$ dimensional vector $\mathbf{z}$. Given a mapping $\mathbf{z}=\phi(\mathbf{x})$, to set up our new optimization problem, we simply replace all occurences of $\mathbf{x}$ with $\phi(\mathbf{x})$.

\section{PRINCIPAL COMPONENT ANALYSIS}

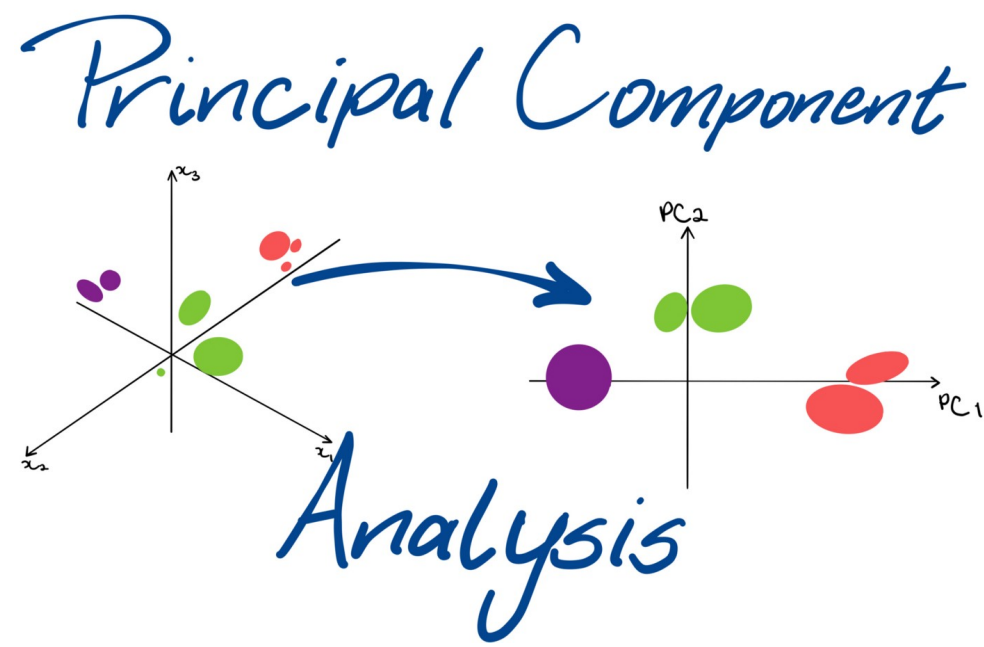

FIG. 3: Principal component analysis. Source: Towards Data science

Principal component analysis (PCA) is a technique for reducing the dimensionality of large datasets, increasing interpretability but at the same time minimizing information loss (Jolliffe and Cadima (2016); Shlens (2014), also see Fig. 3). It does so by creating new uncorrelated variables that successively maximize variance. Finding such new variables, the principal components, reduces to solving an eigenvalue/eigenvector problem, and the new variables are defined by the dataset at hand, not a priori, hence making PCA an adaptive data analysis technique. The steps are

$\checkmark$ Standardize the range of continuous initial variables

$\checkmark$ Compute the covariance matrix to identify correlations

$\checkmark$ Compute the eigenvectors and eigenvalues of the covariance matrix to identify the principal components

$\checkmark$ Create a feature vector to decide which principal components to keep

$\checkmark$ Recast the data along the principal components axes

\section{References}

Burges, C.J., A tutorial on support vector machines for pattern recognition, Data mining and knowledge discovery, vol. 2, no. 2, pp. 121-167, 1998 .

Cortes, C. and Vapnik, V., Support-vector networks, Machine learning, vol. 20, no. 3, pp. 273-297, 1995. 
Dana, S., Addressing challenges in modeling of coupled flow and poromechanics in deep subsurface reservoirs, PhD thesis, The University of Texas at Austin, 2018.

Dana, S., System of equations and staggered solution algorithm for immiscible two-phase flow coupled with linear poromechanics, arXiv preprint arXiv:1912.04703, 2019.

Dana, S., Ganis, B., and Wheeler, M.F., A multiscale fixed stress split iterative scheme for coupled flow and poromechanics in deep subsurface reservoirs, Journal of Computational Physics, vol. 352, pp. 1-22, 2018.

Dana, S., Ita, J., and Wheeler, M.F., The correspondence between voigt and reuss bounds and the decoupling constraint in a twogrid staggered algorithm for consolidation in heterogeneous porous media, Multiscale Modeling \& Simulation, vol. 18, no. 1, pp. 221-239, 2020.

Dana, S. and Jha, B., A fault slip model to study earthquakes due to pore pressure perturbations, arXiv preprint arXiv:2104.06257, 2021.

Dana, S. and Wheeler, M.F., Convergence analysis of fixed stress split iterative scheme for anisotropic poroelasticity with tensor biot parameter, Computational Geosciences, vol. 22, no. 5, pp. 1219-1230, $2018 \mathrm{a}$.

Dana, S. and Wheeler, M.F., Convergence analysis of two-grid fixed stress split iterative scheme for coupled flow and deformation in heterogeneous poroelastic media, Computer Methods in Applied Mechanics and Engineering, vol. 341, pp. 788-806, 2018b.

Dana, S. and Wheeler, M.F., Design of convergence criterion for fixed stress split iterative scheme for small strain anisotropic poroelastoplasticity coupled with single phase flow, arXiv preprint arXiv:1912.06476, 2019.

Dana, S., Zhao, X., and Jha, B., Two-grid method on unstructured tetrahedra: Applying computational geometry to staggered solution of coupled flow and mechanics problems, arXiv preprint arXiv:2102.04455, 2021.

Foulger, G.R., Wilson, M.P., Gluyas, J.G., Julian, B.R., and Davies, R.J., Global review of human-induced earthquakes, EarthScience Reviews, vol. 178, pp. 438-514, 2018.

Jolliffe, I.T. and Cadima, J., Principal component analysis: a review and recent developments, Philosophical Transactions of the Royal Society A: Mathematical, Physical and Engineering Sciences, vol. 374, no. 2065, p. 20150202, 2016.

Krizhevsky, A., Sutskever, I., and Hinton, G.E., Imagenet classification with deep convolutional neural networks, Advances in neural information processing systems, vol. 25, pp. 1097-1105, 2012.

McCann, M.T., Jin, K.H., and Unser, M., Convolutional neural networks for inverse problems in imaging: A review, IEEE Signal Processing Magazine, vol. 34, no. 6, pp. 85-95, 2017.

Shlens, J., A tutorial on principal component analysis, arXiv preprint arXiv:1404.1100, 2014.

Valentine, A.P. and Trampert, J., Data space reduction, quality assessment and searching of seismograms: autoencoder networks for waveform data, Geophysical Journal International, vol. 189, no. 2, pp. 1183-1202, 2012.

Yoon, C.E., O’Reilly, O., Bergen, K.J., and Beroza, G.C., Earthquake detection through computationally efficient similarity search, Science advances, vol. 1, no. 11, p. e1501057, 2015. 